\title{
Effects of new physics in neutrino oscillations in matter
}

\author{
Mario Campanelli ${ }^{a}$ and Andrea Romanino ${ }^{b}$ \\ ${ }^{a}$ Departement de Physique Nucleaire et Corpuscolaire, Université de Genève \\ CH-1211 Genève 4, Switzerland \\ ${ }^{b}$ Scuola Normale Superiore and INFN, Sezione di Pisa, \\ I-56126 Pisa, Italy
}

\begin{abstract}
A new flavor changing electron neutrino interaction with matter would always dominate the $\nu_{e}$ oscillation probability at sufficiently high neutrino energies. Being suppressed by $\theta_{13}$, the energy scale at which the new effect starts to be relevant may be within the reach of realistic experiments, where the peculiar dependence of the signal with energy could give rise to a clear signature in the $\nu_{e} \rightarrow \nu_{\tau}$ channel. The latter could be observed by means of a coarse large magnetized detector by exploiting $\tau \rightarrow \mu$ decays. We discuss the possibility of identifying or constraining such effects with a high energy neutrino factory. We also comment on the model independent limits on them.
\end{abstract}

\section{Introduction}

The Standard Model (SM) provides an elegant minimal framework for studying neutrino physics. In this framework, neutrinos are produced and detected, and interact with matter during propagation, through charged and neutral current renormalizable interactions. At the same time, the mixing with charged leptons and the (small) masses can be accounted for by non-renormalizable interactions in the form $h_{i j}\left(L_{i} H\right)\left(L_{j} H\right) / \Lambda$. Whatever is the ultraviolet completion of the SM, it would be no surprise if the direct and indirect signals looked for at colliders and low-energy experiments were accompanied by effects in neutrino experiments. Indeed, such effects may arise at a significant level e.g. in supersymmetric [1] or extra-dimensional [2, 3] new physics scenarios.

The possibility that new physics (NP) affects the neutrino transitions observed in solar [4, 5, 6, [7, atmospheric [8, 9, 10], LSND [11], and supernova [12] experiments has been widely studied in the literature. Here we consider, from a model independent point of view, the possibility of measurable effects in a high intensity controlled neutrino experiment. As in all other cases, NP can give rise to corrections to the production or detection interaction [13, 14, 15], as well as to the interaction with matter during propagation [16, 17, 15, 18]. Here we are interested in the latter possibility. Effects in the production and detection processes may also play a role, although not necessarily. However, the features of the two types of effects are quite different, which should make relatively easy to disentangle them. In fact, due to the geometrical $L^{-2}$ suppression, the latter are best studied at a smaller baseline $L$ [14, whereas in the former case the $L^{-2}$ suppression is compensated (up to a certain $L$ ) by the unfolding of the oscillation. Moreover, the former effect exhibits a peculiar growth with the energy which, as we will see, may give rise to a noticeable signature. 
New (coherent) effects during propagation manifest themselves as an effective potential contributions to the neutrino squared mass matrix $M^{2}$, the potentially largest one contributing to $M_{\nu_{e} \nu_{\tau}}^{2}$ [5, 10, 6, 7]. Due to the large $\nu_{\mu}-\nu_{\tau}$ mixing $\left(\theta_{23}\right)$, the latter will affect both $\nu_{e} \rightarrow \nu_{\mu}$ and $\nu_{e} \rightarrow \nu_{\tau}$ oscillations. However, the effect in the $\nu_{e} \rightarrow \nu_{\tau}$ channel is energy enhanced, while the one in the $\nu_{e} \rightarrow \nu_{\mu}$ is not. As a consequence, the $\nu_{\tau}$ spectrum may have a striking enhancement at high energy contrary to the conventional MSW prediction. Direct event-by-event detection of such an effect (as well as detection of effects in the $\nu_{\mu} \rightarrow \nu_{\tau}$ channel) [16] would require a very granular detector for $\tau$ identification. Would a coarse neutrino detector with only muon charge identification capability miss the peculiar feature of the signal and possibly confuse it with a pure oscillation signal (or viceversa)? Not completely, since the $\nu_{e} \rightarrow \nu_{\tau}$ channel will contribute to the wrong sign muon spectrum through $\tau \rightarrow \mu$ decay. This has also implications for the sensitivity of a wrong sign experiment to new physics, which becomes strongly dependent on the energy. Such an unequivocal departure from MSW predictions would represent a clean signal and a handle to separate the effect from standard oscillations or corrections to the production or detection interaction. Another effect, not considered here since it would require a detector with ability of distinguishing between electron-like and neutral current-like events, would be a large increase of the latter sample, due to hadronic tau decays.

\section{Theoretical background}

The standard MSW effect gives rise to diagonal contributions to the neutrino squared mass matrix proportional to the neutrino energy. At energies above the resonance, those terms suppress the electron neutrino mixing. On the other hand, a flavor changing neutrino interaction would give rise to a non-diagonal term which, although smaller than the diagonal ones, will also grow with the neutrino energy. In the high energy limit, the two matter induced terms will eventually dominate the mass matrix. Unlike in the conventional MSW case, where the mixing angle goes to zero, in this case the mixing angle reaches an asymptotic value which measures the ratio between the flavor changing and flavor conserving interactions.

The interest of this simple observation depends on the scale at which the new interaction starts to become relevant. This happens when the new matter term becomes comparable to the original entry in the squared mass matrix. Particularly interesting is then a $\nu_{e}-\nu_{\tau}$ flavor changing interaction. The original $M_{\nu_{e} \nu_{\tau}}^{2}$ term, as well as $M_{\nu_{e} \nu_{\mu}}^{2}$, is in fact suppressed by $\theta_{13}$, the only neutrino mixing angle which is certainly not large. At the same time, the bounds on $\nu_{e}-\nu_{\tau}$ interactions are the weakest among all possible neutrino flavor changing interactions.

Let us discuss the points above in greater detail. The neutrino potential induced by $\nu_{\alpha} f \rightarrow \nu_{\beta} f$ interactions $(\alpha, \beta=e, \mu, \tau$ and $f$ represents an electron or a up or down quark) can be parameterized as $V_{\alpha \beta}=\sqrt{2} G_{F} \epsilon_{\alpha \beta} N_{e}=\epsilon_{\alpha \beta} V$, where $V=\sqrt{2} G_{F} N_{e}, N_{e}$ is the electron number density and $\epsilon_{\alpha \beta}=$ $\left|\epsilon_{\alpha \beta}\right| e^{i \phi_{\alpha \beta}}$ are small parameters satisfying $\epsilon_{\beta \alpha}=\epsilon_{\alpha \beta}^{*}$ (see the Appendix for a detailed discussion of the bounds on these parameters).

The effective neutrino squared mass matrix $M_{\mathrm{eff}}{ }^{2}$ is then

$$
M_{\mathrm{eff}}^{2}=U\left(\begin{array}{ccc}
0 & 0 & 0 \\
0 & \Delta m_{21}^{2} & 0 \\
0 & 0 & \Delta m_{31}^{2}
\end{array}\right) U^{\dagger}+2 E V\left(\begin{array}{ccc}
1+\epsilon_{e e} & \epsilon_{e \mu} & \epsilon_{e \tau} \\
\epsilon_{\mu e} & \epsilon_{\mu \mu} & \epsilon_{\mu \tau} \\
\epsilon_{\tau e} & \epsilon_{\tau \mu} & \epsilon_{\tau \tau}
\end{array}\right),
$$

where $E$ is the neutrino energy, $U$ is the MNS mixing matrix in the usual parameterization and $\Delta m_{21}^{2}>0$ is the smaller squared mass difference.

In order to have an intuitive picture of the basic features of the effects under study, let us first of all set $\Delta m_{21}^{2}=0$. Whereas in this limit the angle $\theta_{12}$ becomes unphysical, in presence of new 
physics the CP-violating phase $\delta$ does not, contrary to what sometimes stated in the literature. In fact, the phase redefinition necessary to rotate $\delta$ away from the mixing matrix moves $\delta$ in the non-diagonal new interactions. In the convention in which the $\epsilon$ parameters are initially real and the mixing matrix is complex], the $\epsilon$ 's become complex once the phase has been rotated away from the mixing matrix.

Having set $\Delta m_{21}^{2}=0$ we can rewrite eq. (11) as

$$
M_{\mathrm{eff}}^{2}=\Delta m_{31}^{2}\left(\begin{array}{ccc}
\left|s_{13}^{2}\right|^{2}+\left(E / E_{\mathrm{res}}\right)\left(1+\epsilon_{e e}\right) & s_{13}^{*} / \sqrt{2}+\left(E / E_{\mathrm{res}}\right) \epsilon_{\mu e}^{*} & s_{13}^{*} / \sqrt{2}+\left(E / E_{\mathrm{res}}\right) \epsilon_{\tau e}^{*} \\
s_{13} / \sqrt{2}+\left(E / E_{\mathrm{res}}\right) \epsilon_{\mu e} & 1 / 2+\left(E / E_{\mathrm{res}}\right) \epsilon_{\mu \mu} & 1 / 2+\left(E / E_{\mathrm{res}}\right) \epsilon_{\tau \mu}^{*} \\
s_{13} / \sqrt{2}+\left(E / E_{\mathrm{res}}\right) \epsilon_{\tau e} & 1 / 2+\left(E / E_{\mathrm{res}}\right) \epsilon_{\tau \mu} & 1 / 2+\left(E / E_{\mathrm{res}}\right) \epsilon_{\tau \tau}
\end{array}\right),
$$

where we also set $\theta_{23}=\pi / 4, \cos \theta_{13}, \cos 2 \theta_{13}=1$ and we denoted $s_{13} \equiv \sin \theta_{13} e^{i \delta}$. The resonant energy $E_{\text {res }}$ is

$$
E_{\mathrm{res}} \simeq 10 \mathrm{GeV}\left(\frac{\Delta m_{31}^{2}}{2.5 \cdot 10^{-3} \mathrm{eV}^{2}}\right)\left(\frac{1.65 \mathrm{~g} \mathrm{~cm}^{3}}{\rho Y_{e}}\right)
$$

where $Y_{e}$ is the number of electrons per baryon in matter $n_{e} / n_{B}$. The $E / E_{\text {res }}$ enhancement of matter effects allows even small $\epsilon$ parameters to have a role at sufficiently high energy. This may happen at accessible energies especially in the case of the $\epsilon_{\tau e}$ parameter, which is most weekly constrained - values of $\epsilon_{\tau e}$ as large as 0.1 are not excluded, see the Appendix. In fact, for $\epsilon_{\tau e}=0.1$ and $E=50 \mathrm{GeV}$, the NP term in $\left(M_{\text {eff }}\right)_{\tau e}$ would correspond to a maximal $\sin ^{2} 2 \theta_{13}$. In general, $\epsilon_{\tau e}$ has an effect comparable to that of a $\sin \theta_{13} \simeq 7 \epsilon(E / 50 \mathrm{GeV})$. Since machines with a sensitivity to $\sin \theta_{13}$ as low as $0.5 \cdot 10^{-2}$ are conceivable, we conclude that sensitivities well below $\epsilon_{\tau e}=0.1$ are in principle achievable. Of course, the standard oscillation effect could still be larger and hide the new effect. However, the $\epsilon_{\tau e}$ term, as the $\epsilon_{\tau \mu}$ one, has the additional merit of contributing to an entry of $M_{\text {eff }}$ that is suppressed by $\theta_{13}$ is absence of new physics. Therefore, the $\epsilon_{\tau e}$ term becomes comparable to and then exceeds the standard one for energies $E \gtrsim E_{\mathrm{NP}}$, where $E_{\mathrm{NP}}=\left|s_{13} /(\sqrt{2} \epsilon)\right| E_{\text {res }}$ is suppressed by $\theta_{13}$. The regime where the new effects are comparable to the standard ones is within the reach of a machine producing neutrinos of maximum energy $E_{\max }$ if

$$
|\epsilon| \gtrsim \frac{\left|s_{13}\right|}{\sqrt{2}} \frac{E_{\mathrm{res}}}{E_{\max }}
$$

For example, for $E_{\max }=50 \mathrm{GeV}$, that condition becomes $|\epsilon| \gtrsim 0.007\left(\left|s_{13}\right| / 0.05\right)$. We recall that $\left|s_{13}\right|=0.05$ corresponds to $\sin ^{2} 2 \theta_{13}=10^{-2}$, a value one order of magnitude below the present bound and well within a typical sensitivity.

From the experimental point of view, a study of the effect in the $\nu_{e} \leftrightarrow \nu_{\tau}$ channel through direct $\tau$ detection is certainly challenging. However, it is possible to take advantage of $\tau$ decays into muons to look for the effect in the spectrum of "wrong sign" muon events by means of a coarse neutrino detector with only muon identification capabilities (see e.g. [19]). Whereas a sizable $\epsilon_{\tau \mu}$ might also give rise to some effects, we focus here on $\epsilon_{\tau e}$ and set all the other $\epsilon_{\alpha \beta}$ parameters to zero in our numerical calculations.

Let us now discuss in greater detail the high energy enhancement of the $\nu_{\tau}$ spectrum in presence of a sizable $\epsilon_{\tau e}$ and see that there is not a corresponding enhancement of the $\nu_{\mu}$ spectrum, despite the large mixing of $\nu_{\mu}$ and $\nu_{\tau}$. To this aim let us consider the leading terms of the oscillation

\footnotetext{
${ }^{1}$ The parameterization choice for the MNS matrix fixes the phase convention for the $\epsilon$ parameters as well.
} 
probabilities in the high energy limit $E \gg E_{\text {res }}$ and compare the cases with and without new physics.

In the standard case (and in the $\Delta m_{21}^{2}=0$ limit) $\theta_{12}$ is unphysical and the $\theta_{23}$ rotation commutes with the matter term, so matter effects only modify $\theta_{13}$. Moreover, the $\nu_{e} \leftrightarrow \nu_{\tau}\left(\nu_{e} \leftrightarrow \nu_{\mu}\right)$ oscillation probability coincides with a two neutrino oscillation probability in matter with vacuum amplitude $\cos ^{2} \theta_{23} \sin ^{2} 2 \theta_{13}\left(\sin ^{2} \theta_{23} \sin ^{2} 2 \theta_{13}\right)$ and squared mass difference $\Delta m_{31}^{2}$. In the limit $E \gg E_{\text {res }}$, the squared mass difference in matter $\Delta m_{31}{ }^{\prime} \sim 2 E V$ grows with energy, canceling the $1 / E$ dependence in the oscillating term of the probability. However, the $\theta_{13}$ mixing angle gets suppressed by the large diagonal MSW term, $\sin ^{2} 2 \theta_{13}^{\prime} \sim \sin ^{2} 2 \theta_{13}\left(E_{\text {res }} / E\right)^{2}$, so the transition probabilities still decrease as $E^{2}$,

$$
\begin{aligned}
& P\left(\nu_{e} \rightarrow \nu_{\tau}\right) \sim\left(\frac{E_{\mathrm{res}}}{E}\right)^{2} \cos ^{2} \theta_{23} \sin ^{2} 2 \theta_{13} \sin ^{2} \frac{L V}{2} \\
& P\left(\nu_{e} \rightarrow \nu_{\mu}\right) \sim\left(\frac{E_{\mathrm{res}}}{E}\right)^{2} \sin ^{2} \theta_{23} \sin ^{2} 2 \theta_{13} \sin ^{2} \frac{L V}{2} .
\end{aligned}
$$

In presence of $\nu_{e}-\nu_{\tau}$ or $\nu_{e}-\nu_{\mu}$ flavor changing interactions, the $\theta_{23}$ rotation does not commute with the matter term anymore. As a consequence, the oscillation probability in the $\Delta m_{21}^{2}=0$ limit has not a simple two neutrino form. Two squared mass differences enter the oscillation probabilities, $\Delta m_{31}^{2}$ and $2 E V$ in the large $E / E_{\text {res }}$ limit. Moreover, whereas the $\theta_{23}$ is essentially not affected, $s_{13}$ becomes $s_{13}^{\prime} \simeq E / E_{\mathrm{res}} s_{13}+c_{23} \epsilon_{\tau e}+s_{23} \epsilon_{\mu e}$ and a non vanishing (and physical) 12 angle is generated $\theta_{12}^{\prime} \simeq-s_{23} \epsilon_{\tau e}+c_{23} \epsilon_{\mu e}$. Approximate analytical formulae for the oscillation probabilities are unfortunately quite cumbersome. At the moment, however, we are only interested to the leading contributions in the large $E / E_{\text {res }}$ limit, that have the following simple expression,

$$
\begin{aligned}
& P\left(\nu_{e} \rightarrow \nu_{\tau}\right) \sim 4\left|\epsilon_{\tau e}+\frac{E_{\mathrm{res}}}{E} c_{23} s_{13}\right|^{2} \sin ^{2} \frac{L V}{2} \\
& P\left(\nu_{e} \rightarrow \nu_{\mu}\right) \sim 4\left|\epsilon_{\mu e}+\frac{E_{\mathrm{res}}}{E} s_{23} s_{13}\right|^{2} \sin ^{2} \frac{L V}{2},
\end{aligned}
$$

where we included the leading $E_{\mathrm{res}} / E$ correction to the energy-independent amplitudes. We explicitly see that the oscillation probability is not suppressed any more by two powers of the energy but reaches instead a constant value $4|\epsilon|^{2} \sin ^{2}(L V / 2)$ at high energies. In an experiment at a Neutrino Factory, the effect of such an energy independent probability is enhanced by the growth with energy of the neutrino flux and of the neutrino cross section. This gives rise to a striking (approximately cubic before approaching the energy cut off) growth of the signal with energy.

Since $s_{13}=\sin \theta_{13} e^{i \delta}$ and $\epsilon=|\epsilon| e^{i \phi}$, we also see from eqs. (6) that in the high energy limit the amplitude depends on the cosine of the phase difference $\delta-\phi$. Let us consider a CP-conserving case, so that both $s_{13}$ and $\epsilon$ can be made real. Then if the two parameters have the same sign $(\delta-\phi=0)$, the contributions to the amplitude will interfere constructively in the neutrino channel and destructively in the antineutrino one. Viceversa, if the signs are opposite $(\delta-\phi=\pi)$, the interference will be destructive for neutrinos and constructive for antineutrinos. The destructive interference will be maximal for $E \sim E_{\mathrm{NP}}=\left|s_{13} /(\sqrt{2} \epsilon)\right| E_{\mathrm{res}}$, the energy at which the two contributions to the amplitude are comparable. The latter formula and previous considerations hold provided that $E_{\mathrm{NP}}>E_{\mathrm{res}}$, or $\left|s_{13} /(\sqrt{2} \epsilon)\right|>1$, the condition for eqs. (6) to be valid at the energy at which the amplitude vanishes. For $E_{\mathrm{NP}} \lesssim E_{\text {res }}$ the cancellation will be spoiled by the $\Delta m_{31}^{2}$ terms.

Eqs. (6) show that $\epsilon_{\tau e}$ only affects $P\left(\nu_{e} \rightarrow \nu_{\tau}\right)$ at the leading order in $E_{\text {res }} / E$. Since $\nu_{\tau}$ and $\nu_{\mu}$ are largely mixed, $\epsilon_{\tau e}$ also enters $\nu_{e} \leftrightarrow \nu_{\mu}$ oscillations but only through subleading terms omitted in eq. (6] $)$. This is because the mixing takes place at atmospheric squared mass difference $\Delta m_{31}^{2}$, 
which in the large $E_{\text {res }} / E$ high limit is subleading compared to the other squared mass difference $2 E V$.

\section{Oscillation probabilities}

We can show now some examples of how the oscillation probability gets modified by the presence of this new interaction. For doing that we have to consider a specific case. Let us consider then a model with no CP violation and $s_{13}, \epsilon_{\tau e}>0$, 2 with oscillation parameters $\theta_{23}=\pi / 4, \sin ^{2} 2 \theta_{12}=0.08$, $\Delta m_{31}^{2}=3 \times 10^{-3}, \Delta m_{21}^{2}=5 \times 10^{-5}$ and $\sin ^{2} 2 \theta_{13}=0.001$, about ten times smaller than the present bound. It has to be noticed that the smaller the value of $\sin ^{2} 2 \theta_{13}$, the more visible new physics effects are.

We consider a Neutrino Factory with $10^{21}$ muon decays, and a 40 kton detector with only muon identification capabilities, located at a distance of $3000 \mathrm{~km}$ from the accelerator.

Present data on Flavor Changing Interactions suggest bounds on the various elements of the FCI matrix as $\left|\epsilon_{e \mu} \lesssim 7 \times 10^{-5}\right|,\left|\epsilon_{\mu \tau} \lesssim 5 \times 10^{-2}\right|,\left|\epsilon_{e \tau} \lesssim 7 \times 10^{-2}\right|$. Even if we assume that $\epsilon_{\mu e}$ is as large as the experimental bound, its effect on oscillation probabilities will be negligible. An $\epsilon_{\tau \mu}$ at the experimental bound would give rise to non-negligible effects [16] but not to the high-energy enhancement we are focusing on, so we set $\epsilon_{\tau \mu}=0$. The effect of a sizable $\epsilon_{\tau e}$ is shown in Fig. 2, where the solid histograms represent normal matter oscillations whereas the dashed histogram is the new physics case. Assuming $\epsilon_{\tau e}$ is close to the allowed maximum value, the oscillation probability gets modified in quite a dramatic way.

We see that, while the normal oscillation probability decreases like $1 / E_{\nu}^{2}$, in the case of new physics the probability tends to a constant; the difference is of course enhanced at high energy. For antineutrinos, we observe the same behavior at high energy, but we note a difference at intermediate energies $E \sim E_{\mathrm{NP}}$. There, the two terms in the amplitude of eq. (6/a) are comparable and their relative sign is opposite for neutrinos and antineutrinos. The effect of such a sign change is clearly visible but cannot accounted for by eq. (6a) for small values of $s_{13} /\left(\sqrt{2} \epsilon_{\tau e}\right)$, as in the present example. On the contrary, for $s_{13} / \epsilon_{\tau e}>\sqrt{2}$ (and e.g. no CP-violation, $s_{13}, \epsilon_{\tau e}$ both positive as in this example) the sign change would lead to a suppression of the antineutrino probability located at $E \simeq E_{\mathrm{NP}}$, as apparent from eq. (6a). In the latter case, the suppression of the antineutrino oscillation probability measures $s_{13} / \epsilon_{\tau e}=\sqrt{2} E_{\mathrm{NP}} / E_{\mathrm{res}}$. In any case, the difference between the two $\mathrm{CP}$-conjugated channels at $E \sim E_{\mathrm{NP}}$ represents a powerful tool to constrain the relative phase of $s_{13}$ and $\epsilon_{\tau e}$.

In the $\nu_{e} \rightarrow \nu_{\mu}$ channel, the effect of $\epsilon_{\tau e}$ is quite different, as discussed in Sec. 2. At high energy, where the dominant squared mass difference is $2 E V$, the oscillation probabilities are not enhanced. On the contrary, the spectrum does not differ from the pure oscillation case apart from the normalization and falls with energy as in the standard MSW case. The new physics term behaves as a larger $\sin ^{2} 2 \theta_{13}[17,18]$.

\section{Observable effects at a Neutrino Factory}

The neutrino energy distribution in muon decays is the following:

$$
\begin{gathered}
\frac{d^{2} N_{\nu_{\mu}}}{d x d \Omega} \propto \frac{2 x^{2}}{4 \pi}\left[(3-2 x)+(1-2 x) P_{\mu} \cos \theta\right] \\
\frac{d^{2} N_{\bar{\nu}_{e}}}{d x d \Omega} \propto \frac{12 x^{2}}{4 \pi}\left[(1-x)+(1-x) P_{\mu} \cos \theta\right]
\end{gathered}
$$

\footnotetext{
${ }^{2}$ The parameter $s_{13}$ can always been made positive. Then if CP is conserved $\epsilon_{\tau e}$ is real but can have both signs.
} 

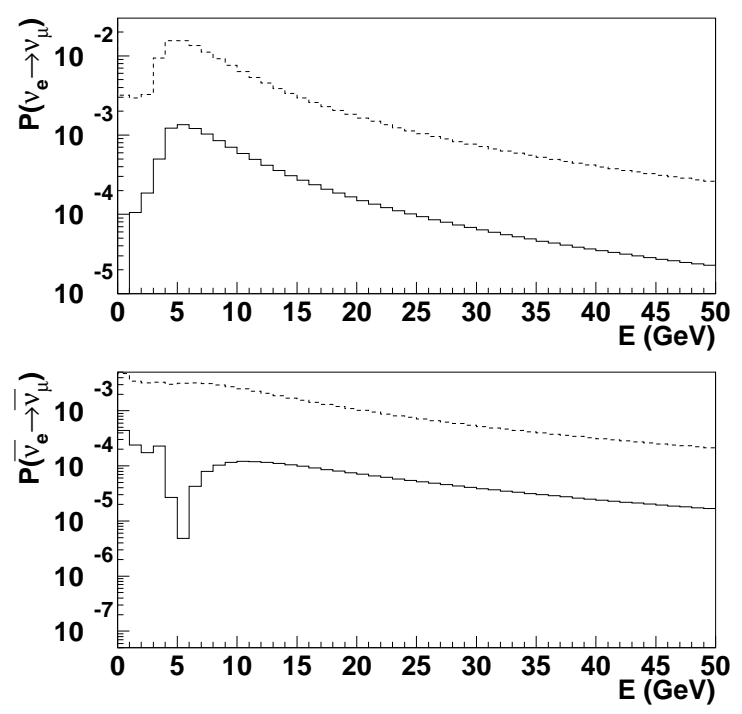

Figure 1: $\nu_{e} \rightarrow \nu_{\mu}$ oscillation probability in the standard MSW model (full line) and in the presence of flavor-changing interactions (dashed line), for $\sin ^{2} 2 \theta_{13}=0.001$ and $\epsilon_{\tau e}=$ 0.07 .
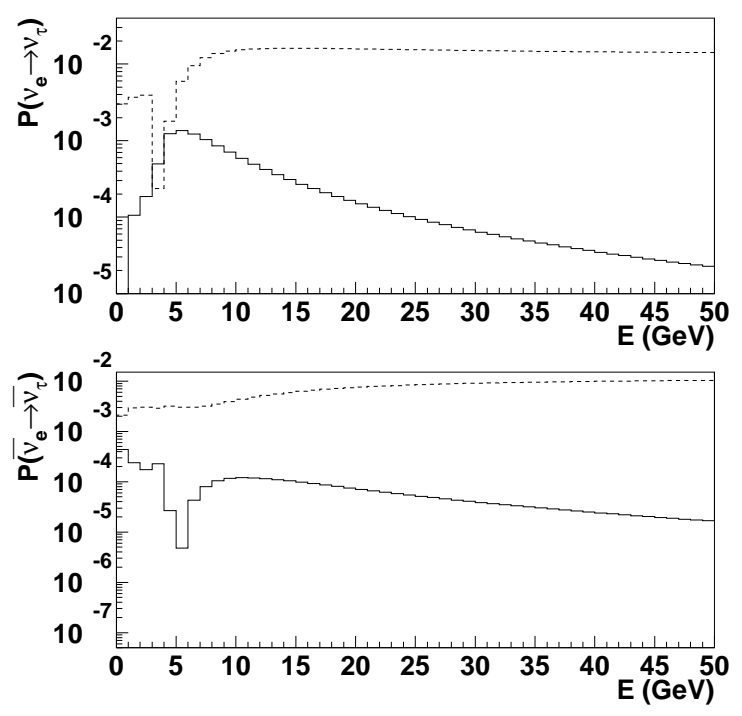

Figure 2: $\nu_{e} \rightarrow \nu_{\tau}$ oscillation probability in the standard MSW model (full line) and in the presence of flavor-changing interactions (dashed line), for $\sin ^{2} 2 \theta_{13}=0.001$ and $\epsilon_{\tau e}=$ 0.07 .

where $x=2 E_{\nu} / m_{\mu}, P_{\mu}$ is the muon polarization, and $\theta$ is the angle between the muon polarization vector and the neutrino direction. In the laboratory frame, the shape of the energy distribution is preserved if a Lorentz boost is applied, so if muons are accelerated to an energy $E_{\mu}$, the spectral shape will be the same, with this time $x=E_{\nu} / E_{\mu}$. The cleanest experimental observable to measure neutrino oscillation is the appearance of wrong-sign muons, i.e. muons observed in the detector with a charge opposite to those circulating in the storage ring. When positive muons circulate in the ring, electron neutrinos are produced via

$$
\mu^{+} \rightarrow e^{+} \bar{\nu}_{\mu} \nu_{e}
$$

$\nu_{e}$ would then oscillate into $\nu_{\mu}$, seen as negative (wrong-sign) muons in a far detector. Also $\nu_{e} \rightarrow \nu_{\tau}$ oscillations will contribute to this channel, via $\tau \rightarrow \mu$ decays (B.R. $\approx 17 \%$ ), that will produce muons of the same sign of those coming directly from $\nu_{e} \rightarrow \nu_{\mu}$ oscillations. We already saw in the previous section that the presence of new physics in matter propagation could lead to a noticeable change of the $\nu_{e} \rightarrow \nu_{\tau}$ oscillation probability for large neutrino energies, especially for small values of $\theta_{13}$. This effect will then be visible in the wrong-sign muon spectrum due to $\tau \rightarrow \mu$ decays.

The fact that the neutrino factory flux increases for large energies allows a precise exploration of the high-energy oscillation probability. In figures 3 and 4 we show spectra for wrong-sign muon events in a neutrino factory with $10^{21}$ muon decays and a $40 \mathrm{kt}$ far detector. The large difference observable between normal matter propagation and FCI at high energy is almost only due to $\tau$ decays, since the effect of $\epsilon_{\tau e}$ on $\nu_{e} \rightarrow \nu_{\mu}$ oscillations is is smaller than in $\nu_{e} \rightarrow \nu_{\tau}$ and significant only at intermediate energies, and since the very strong bound existing on $\left|\epsilon_{e \mu}\right|$ prevents any measurable effect from this parameter.

In presence of new effects, one wonders whether it is possible to measure both the new physics 

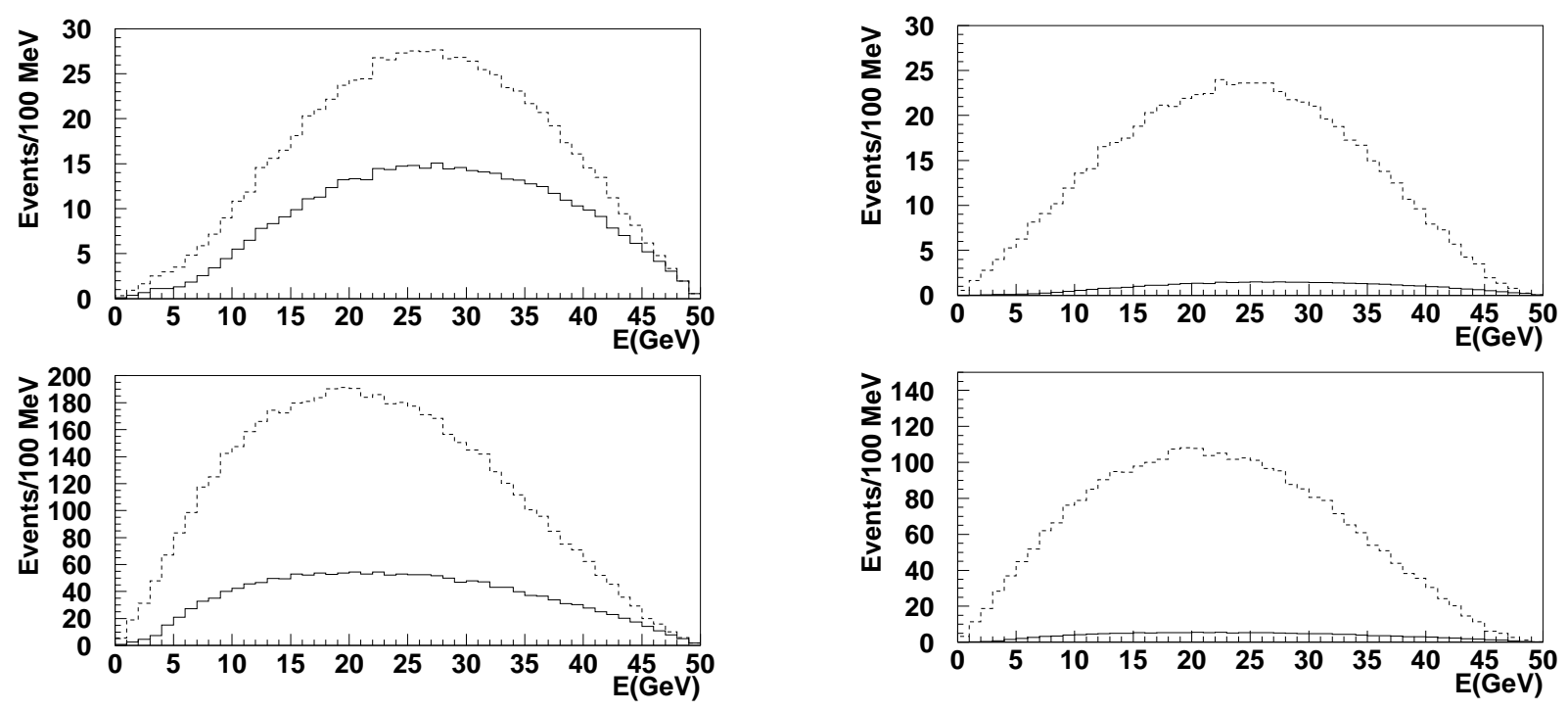

Figure 3: Observed visible energy spectrum for wrong-sign muon events in the case of $\mu^{-}$(upper plot) and $\mu^{+}$(lower plot) circulating in the storage ring. Full histogram is the standard MSW case, dashed histogram is in the presence of new interactions. For the considered value of $\sin ^{2} 2 \theta_{13}=10^{-2}$, the magnitudes of the two effects are similar.

Figure 4: Same plot as before, but considering a value of $\sin ^{2} 2 \theta_{13}=10^{-3}$. As expected, the standard MSW effect (full line) is much reduced, therefore the relative importance of the new physics interaction (dashed line, almost unchanged with respect to the previous case) is largely enhanced. The two plots correspond to $\mu^{-}$and $\mu^{+}$in the ring.

parameters, in our case $\epsilon_{\tau e}$, and the oscillation parameters, in particular $s_{13}$. The spectral information is essential for this purpose. Only counting the number of wrong-sign muons will lead to the impossibility of correctly interpreting the observed neutrino transitions in the case of new physics. The role of $\tau \rightarrow \mu$ decays is crucial here. If they were neglected and only direct $\nu_{e} \rightarrow \nu_{\mu}$ transitions were considered, a confusion would arise between $\epsilon_{\tau e}$ and $\theta_{13}$ even if the spectral information were taken into account [17, 18].

To be more general, figure 5 shows $90 \%$ C.L. contours for observing FCI effects for values of $\left|\epsilon_{e \mu}\right|$ in the range $10^{-4}-10^{-2}$, and $\sin ^{2} 2 \theta_{13}$ between $5 \times 10^{-5}-10^{-2}$.

To produce this plot we consider an experiment observing a certain number of wrong-sign muons in the detector. It is well-known that $\theta_{13}$ has quite a small effect on the energy distribution, therefore can be extracted from a simple counting of the events. The other relevant parameters will be measured with very good precision from the "dip" of the disappearance of right-sign muons (not much affected by new physics effects). The experiment makes a "measurement" of $\theta_{13}$ based on event counting, assuming that no new physics is present, and produces an expected energy spectrum for that particular value of $\theta_{13}$. Then the spectrum without new physics is compared with that actually observed, and a bin-by-bin $\chi^{2}$ is produced. If the $\chi^{2}$ probability of the two histograms to be equal is too small, we consider that no confusion can arise between new physics effects and normal oscillations.

In obtaining figure 5 we have set the new CP-violating phase $\delta-\phi$ to zero. One wonders whether the fact that $\delta-\phi$ is actually unknown would spoil the results in the general case. We 


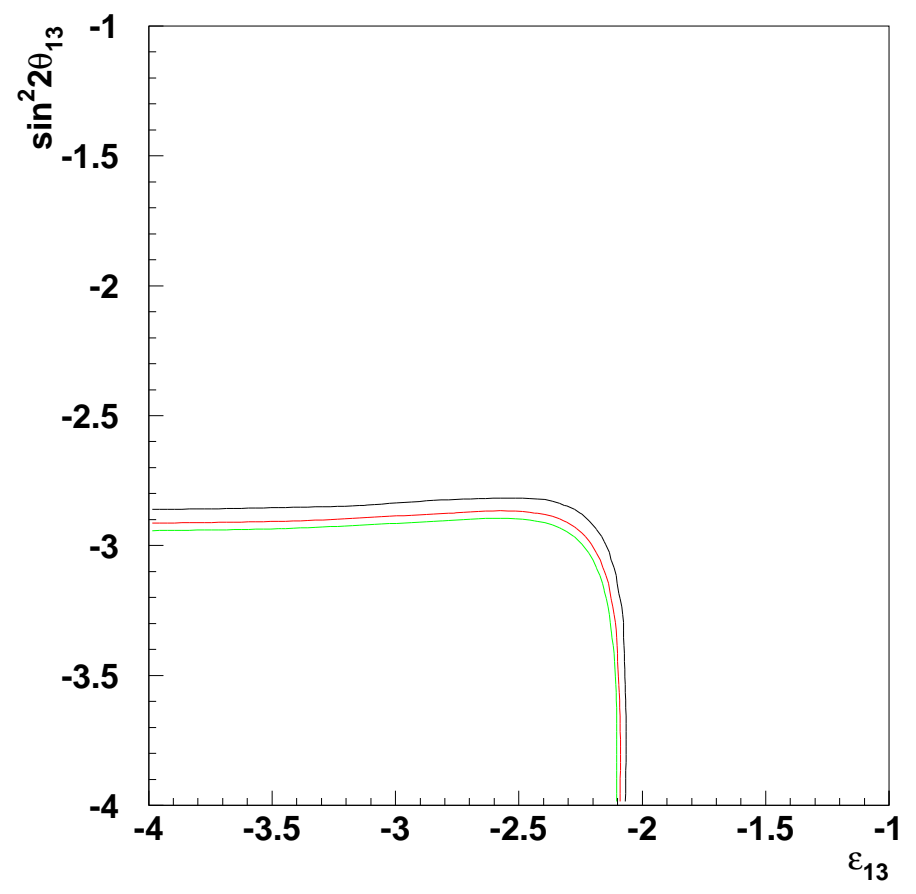

Figure 5: $90 \%$ C.L contour in the plane $\sin ^{2} 2 \theta_{13}, \epsilon_{13}$ of the possibility of distinguishing new physics from standard oscillations, extracting the oscillation parameter $\theta_{13}$ from the counting of the number of events and performing a $\chi^{2}$ test on the spectral shape.

expect that this is not the case provided that data with both $\mu^{+}$and $\mu^{-}$circulating in the ring are used. In fact, as mentioned in the previous section, the comparison of the two sets of data allows in principle to determine the relative sign of $s_{13}$ and $\epsilon_{\tau e}$ and, more generally, the new CP-violating phase $\delta-\phi$. Here, we have implicitly assumed that the information from the comparison of the two sets of data has been taken into account to constrain $\delta-\phi$ and we have hence used only one set for discussing the measurement of $s_{13}$ and $\epsilon_{\tau e}$. A detailed study of CP-violation in this framework is beyond the scope of this paper.

\section{Conclusions}

We considered here the effects of a possible new flavor-changing interaction affecting the propagation of electron neutrinos in matter. Contrary to new effects in neutrino production or interaction, already well constrained by short-baseline experiments, new physics in matter propagation would benefit from very long-baselines, like those considered for a future Neutrino Factory. The characteristic feature of this machine, i.e. the increase of neutrino flux with energy, is also ideal for these studies since new interactions would not suffer from the $1 / E^{2}$ suppression, and therefore be much more visible at high energy. We studied the problem in a quantitative way, assuming a coarse magnetized iron detector, and observed that, for new physics close to the present experimental

\footnotetext{
${ }^{3}$ Notice that the CP-violation effects associated to the new physics phase $\delta-\phi$ have a characteristic energy scale $E \sim E_{\mathrm{NP}}$ whereas the standard CP-violation and other effects related to a $\Delta m_{21}^{2} \neq 0$ are enhanced at low energy. This offers an handle to distinguish the two sources of CP-violation as well.
} 
bounds, a noticeable signal could be observed in $\nu_{e} \rightarrow \nu_{\tau}$ transitions, exploiting the wrong-sign muons produced in $\tau \rightarrow \mu$ decays.

\section{Acknowledgments}

M.C. would like to thank the Scuola Normale of Pisa for the kind ospitality during the last days of writeup of this paper. This work has been partially supported by MIUR and by the EU under TMR contract HPRN-CT-2000-00148.

\section{Appendix}

In this appendix, we review and enlarge the analysis of the limits on the size of NP contributions to $M_{\text {eff }}$. The general form of new four fermion operators leading to additional contributions to the neutrino coherent scattering with an ordinary medium is

$$
\sum_{\substack{f=e, u, d \\ \alpha, \beta=e, \mu, \tau}} 4 \frac{G_{F}}{\sqrt{2}} \bar{\nu}_{\alpha} \gamma^{\mu} \nu_{\beta}\left(\epsilon_{\alpha \beta}^{f_{L}} \bar{f}_{L} \gamma_{\mu} f_{L}+\epsilon_{\alpha \beta}^{f_{R}} \bar{f}_{R} \gamma_{\mu} f_{R}\right)
$$

Needless to say, eq. (7) is written in a basis in which the kinetic term is canonical and the charged fermion masses are diagonal. The $\epsilon$ parameters in eq. (1) are then given by in terms of the $\epsilon^{f}$ in the equation above by

$$
\epsilon=\epsilon^{e}+2 \epsilon^{u}+\epsilon^{d}+\frac{n_{n}}{n_{e}}\left(2 \epsilon^{d}+\epsilon^{u}\right)
$$

where $n_{e}$ and $n_{n}$ are the electron and neutron number densities respectively and we have omitted the flavor indexes. Notice that the $\epsilon$ parameters in eq. (11) have a mild dependence on the distance traveled through the $n_{n} / n_{e}$ ratio.

A model independent limit on $\epsilon_{\tau \mu}$ can be inferred from atmospheric neutrino data [9]: $\epsilon_{\tau \mu} \lesssim$ 0.05. Significant limits on the single $\epsilon^{e, u, d_{\mathrm{S}}}$ can be obtained if one assumes that the operators in eq. (]) originate from $\mathrm{SU}(2)_{W}$ invariant operators. Then experimental bounds on charged lepton processes imply [5, 10, 6]

$$
\begin{aligned}
& \epsilon_{\mu e}^{e} \lesssim 10^{-6} \\
& \epsilon_{\tau \mu}^{e} \lesssim 3 \cdot 10^{-3} \\
& \epsilon_{\tau e}^{e} \lesssim 4 \cdot 10^{-3} \\
& \epsilon_{\mu e}^{u, d} \lesssim 10^{-5} \\
& \epsilon_{\tau \mu}^{u, d} \lesssim 10^{-2} \\
& \epsilon_{\tau e}^{u, d} \lesssim 10^{-2} \text {. }
\end{aligned}
$$

Since $\mathrm{SU}(2)_{W}$ is broken, the limits above can be evaded. To what extent they can be evaded depends on how the operators eq. (7) are generated and how $\mathrm{SU}(2)_{W}$ breaking enters. The case of the exchange of a $\mathrm{SU}(2)_{W}$ multiplet of bosons with $\mathrm{SU}(2)_{W}$ breaking masses has been considered in [10, 6]. In this case, the operators in eq. (7) are still related to the corresponding charged lepton operators. However, the limits can be relaxed by the $\mathrm{SU}(2)_{W}$ breaking masses by a factor of about 7 without a conflict with the electroweak precision data. Alternatively, the effect of $\mathrm{SU}(2)_{W}$ breaking can be studied through an operator expansion in which the breaking shows up as the vev of the Higgs fields in the higher order operators [20].

In both cases above, the limits are inferred from the charged lepton sector by relating the effects in the two sectors. However, it is possible to generate the neutrino operator in eq. (7) without giving rise to any charged lepton effect through a known mechanism not considered before in this context. In the remainder of this appendix, we discuss the limits on these possible additional contributions to eq. (7) from neutrino physics and see that they cannot evade the limits reviewed above. 
Suppose that some physics (e.g. warped or flat extra dimensions [3]) gives rise to the operator

$$
2 \sqrt{2} \varepsilon_{\alpha \beta} G_{F}\left(H L_{\alpha}\right)^{\dagger} i \hat{\partial}\left(H L_{\beta}\right) .
$$

Since this operator only contribute to the neutrino wave function, bringing the neutrino kinetic term back in canonical form will generate new operators as in eq. (7) but will not generate any operators involving charged leptons only [3]. The couplings in eq. (7) generated through this mechanism are

$$
\begin{gathered}
\epsilon_{\alpha \beta}^{e}=-\frac{1}{2}\left(\varepsilon_{\alpha e} \delta_{\beta e}+\varepsilon_{e \beta} \delta_{\alpha e}\right)+\left(\frac{1}{2}-2 \sin ^{2} \theta_{W}\right) \varepsilon_{\alpha \beta} \\
\epsilon_{\alpha \beta}^{u}=-\left(\frac{1}{2}-\frac{4}{3} \sin ^{2} \theta_{W}\right) \varepsilon_{\alpha \beta} \quad \epsilon_{\alpha \beta}^{d}=\left(\frac{1}{2}-\frac{2}{3} \sin ^{2} \theta_{W}\right) \varepsilon_{\alpha \beta} .
\end{gathered}
$$

Here the $\varepsilon$ parameters are only constrained by neutrino experiments, that give $[3]\left|\varepsilon_{\mu e}\right|<0.05$, $\left|\varepsilon_{\tau e}\right|<0.1,\left|\varepsilon_{\tau \mu}\right|<0.013$. In turn, these bounds imply e.g. $\left|\epsilon_{\mu e}^{e}\right|<0.025,\left|\epsilon_{\tau e}^{e}\right|<0.05$, to be compared with the much more restrictive $\left|\epsilon_{\mu e}^{e}\right|<7 \cdot 10^{-6},\left|\epsilon_{\tau e}^{e}\right|<28 \cdot 10^{-3}$ quoted above. Therefore, the operator in eq. (11) gives the potentially largest contribution to $\epsilon_{\mu e}^{e}, \epsilon_{\tau e}^{e}$. However, what matters in our case is the value of $\epsilon_{\alpha \beta}$ rather than the individual $\epsilon_{\alpha \beta}^{e, u, d}$,

$$
\epsilon_{\alpha \beta}=-\frac{1}{2}\left(\varepsilon_{\alpha e} \delta_{\beta e}+\varepsilon_{e \beta} \delta_{\alpha e}\right)+\frac{1}{2} \frac{n_{n}}{n_{e}} \varepsilon_{\alpha \beta} .
$$

In particular, the contribution to the quantity we are interested in, $\epsilon_{\tau e}=\left(n_{n} / n_{e}-1\right) \varepsilon_{\tau e} / 2$, is suppressed in the earth by the small $\left(n_{n} / n_{e}-1\right) / 2$ factor. Such a suppression makes the corresponding bound weaker than those coming from eqs. (9).

\section{References}

[1] C.S. Aulakh and R.N. Mohapatra, Phys. Lett. B119 (1982) 136.

F. Zwirner, Phys. Lett. B132 (1983) 103.

L.J. Hall and M. Suzuki, Nucl. Phys. B231 (1984) 419.

J.R. Ellis et al., Phys. Lett. B150 (1985) 142.

G.G. Ross and J.W.F. Valle, Phys. Lett. B151 (1985) 375.

R. Barbieri and A. Masiero, Nucl. Phys. B267 (1986) 679.

[2] K.R. Dienes, E. Dudas and T. Gherghetta, Nucl. Phys. B557 (1999) 25, hep-ph/9811428.

N. Arkani-Hamed et al., (1998), hep-ph/9811448.

Y. Grossman and M. Neubert, Phys. Lett. B474 (2000) 361, hep-ph/9912408.

[3] A. De Gouvea et al., Nucl. Phys. B623 (2002) 395, hep-ph/0107156,

[4] L. Wolfenstein, Phys. Rev. D17 (1978) 2369.

J.W.F. Valle, Phys. Lett. B199 (1987) 432.

M. Fukugita and T. Yanagida, Phys. Lett. B206 (1988) 93.

E. Roulet, Phys. Rev. D44 (1991) 935.

M.M. Guzzo, A. Masiero and S.T. Petcov, Phys. Lett. B260 (1991) 154.

V.D. Barger, R.J.N. Phillips and K. Whisnant, Phys. Rev. D44 (1991) 1629.

S. Degl'Innocenti and B. Ricci, Mod. Phys. Lett. A8 (1993) 471.

G.L. Fogli and E. Lisi, Astropart. Phys. 2 (1994) 91.

P.I. Krastev and J.N. Bahcall, Santa Monica 1997, Flavor-changing neutral currents, pp. 259-263, 1997, hep-ph/9703267. 
[5] S. Bergmann, Nucl. Phys. B515 (1998) 363, hep-ph/9707398.

[6] S. Bergmann et al., Phys. Rev. D62 (2000) 073001, hep-ph/0004049.

[7] Z. Berezhiani, R.S. Raghavan and A. Rossi, (2001), hep-ph/0111138.

[8] E. Ma and P. Roy, Phys. Rev. Lett. 80 (1998) 4637, hep-ph/9706309.

G. Brooijmans, (1998), hep-ph/9808498.

M.C. Gonzalez-Garcia et al., Phys. Rev. Lett. 82 (1999) 3202, hep-ph/9809531.

P. Lipari and M. Lusignoli, Phys. Rev. D60 (1999) 013003, hep-ph/9901350.

N. Fornengo et al., Phys. Rev. D65 (2002) 013010, hep-ph/0108043.

[9] N. Fornengo, M.C. Gonzalez-Garcia and J.W.F. Valle, jhep 07 (2000) 006, hep-ph/9906539.

[10] S. Bergmann, Y. Grossman and D.M. Pierce, Phys. Rev. D61 (2000) 053005, hep-ph/9909390.

[11] S. Bergmann and Y. Grossman, Phys. Rev. D59 (1999) 093005, hep-ph/9809524.

[12] S. Mansour and T.K. Kuo, Phys. Rev. D58 (1998) 013012, hep-ph/9711424.

S. Bergmann and A. Kagan, Nucl. Phys. B538 (1999) 368, hep-ph/9803305.

G.L. Fogli et al., (2002), hep-ph/0202269.

[13] A. Bueno et al., JHEP 06 (2001) 032, hep-ph/0010308.

I.I.Y. Bigi et al., (2001), hep-ph/0106177.

NuTeV, J.A. Formaggio et al., Phys. Rev. Lett. 87 (2001) 071803, hep-ex/0104029.

A. Datta et al., Phys. Rev. D64 (2001) 015011, hep-ph/0011375.

[14] M.C. Gonzalez-Garcia et al., Phys. Rev. D64 (2001) 096006, hep-ph/0105159.

[15] T. Ota, J. Sato and N.a. Yamashita, (2001), hep-ph/0112329.

[16] A.M. Gago et al., Phys. Rev. D64 (2001) 073003, hep-ph/0105196.

P. Huber and J.W.F. Valle, Phys. Lett. B523 (2001) 151, hep-ph/0108193.

[17] P. Huber, T. Schwetz and J.W.F. Valle, (2001), hep-ph/0111224.

[18] P. Huber, T. Schwetz and J.W.F. Valle, (2002), hep-ph/0202048.

[19] A. Cervera et al., Nucl. Phys. B579 (2000) 17, hep-ph/0002108.

[20] Z. Berezhiani and A. Rossi, (2001), hep-ph/0111137. 